\title{
ON BLOCH FUNCTIONS AND GAP SERIES
}

\author{
DANIEL GIRELA
}

\begin{abstract}
Kennedy obtained sharp estimates of the growth of the Nevanlinna characteristic of the derivative of a function $f$ analytic and with bounded characteristic in the unit disc. Actually, Kennedy's results are sharp even for VMOA functions. It is well known that any BMOA function is a Bloch function and any VMOA function belongs to the little Bloch space. In this paper we study the possibility of extending Kennedy's results to certain classes of Bloch functions. Also, we prove some more general results obtaining sharp comparison results between the integral means $M_{P}(r, f)$ with $T(r, f)$ for certain classes of functions $f$ analytic in the unit disc.
\end{abstract}

The Nevanlinna class, denoted by $N$, consists of those functions $f$ analytic in the unit disc $U$ for which $\sup _{0<r<1} T(r, f)<\infty$, where $T(r, f)$ denotes the Nevanlinna characteristic of $f$. Kennedy proved that if $f \in N$ then

(1) $\int_{0}^{1}(1-r) \exp \left(2 T\left(r, f^{\prime}\right)\right) d r<\infty$ and $(2) \lim _{r \rightarrow 1}\left(\log \frac{1}{1-r}-T\left(r, f^{\prime}\right)\right)=\infty$.

Both results are sharp. We note that (2) follows from (1).

Let $B$ denote the space of Bloch functions. Two important subspaces of $B$ are those denoted by $B_{0}$ and $B_{1}$. The space $B_{0}$ consists of those $f \in B$ such that $(1-|z|)\left|f^{\prime}(z)\right| \longrightarrow 0$, as $|z| \longrightarrow 1$, and $B_{1}$ consists of those $f \in B$ such that if $\left\{z_{n}\right\} \subset U$ and $\left|f\left(z_{n}\right)\right| \longrightarrow \infty$ then $\left(1-\left|z_{n}\right|\right)\left|f^{\prime}\left(z_{n}\right)\right| \longrightarrow 0$. It is well known that VMOA $\subset B_{0}$ and BMOA $\subset B$. Kennedy's estimates are actually sharp for VMOA functions. In this paper we study the question of whether or not (1) and/or (2) rcmain true for a function $f$ in $B, B_{1}$ or $B_{0}$.

We prove that (2) need not be true for a Bloch function showing that the trivial estimate $T\left(r, f^{\prime}\right) \leq \log \frac{1}{1-r}+O(1)$ is the best that we can say in general. However (2) is true for any $f \in B_{0}$ even though it may not satisfy (1). We do not know whether or not (2) is true for any $f \in B_{1}$ but we can prove that it satisfies (2) with lim sup instead of lim.

Also, we generalize these results obtaining sharp comparison results between the integral means $M_{p}(r, f)$ with $T(r, f)$ for certain classes of functions $f$ anialytic in $U$. 


\section{Introduction and main results}

Let $f$ be a function analytic in the unit disc $U=\{z \in \mathbb{C}:|z|<1\}$. Then, the Nevanlinna characteristic $T(r, f)$ is defined by

$$
T(r, f)=\frac{1}{2 \pi} \int_{-\pi}^{\pi} \log ^{+}\left|f\left(r e^{i t}\right)\right| d t, \quad 0 \leq r<1 .
$$

The Nevanlinna class, denoted by $N$, consists of those $f$ analytic in $U$ for which

$$
\sup _{0 \leq r<1} T(r, f)<\infty
$$

It is well known that $f^{\prime}$ need not belong to $N$ even if $f$ is bounded. This was first proved by Frostman [6] who showed the existence of a Blaschke product whose derivative is not of bounded characteristic. Kennedy determined in [14] as closely as possible the restriction imposed on the growth of $T\left(r, f^{\prime}\right)$ by $(1.2)$. He proved the following two theorems.

Theorem A. ([14, Th. I]). Let $f \in N$. Then

$$
\int_{0}^{1}(I-r) \exp \left(2 T\left(r, f^{\prime}\right)\right) d r<\infty .
$$

Theorem B. ([14, Th. II]). Let $\mu$ be a positive increasing function in $(0,1)$ such that

$$
(1-r) \exp \mu(r) \text { is decreasing. }
$$

$$
\mu(r)-\mu(\rho) \longrightarrow \infty \text { as } \frac{1-r}{1-\rho} \longrightarrow 0
$$

$$
\int_{0}^{1}(1-r) \exp (2 \mu(r)) d r<\infty .
$$

Then there exists $f \in N$ such that

$$
T\left(r, f^{\prime}\right)>\mu(r)
$$

for all $r$ sufficiently close to 1 .

Let us notice that, since $T\left(r, f^{\prime}\right)$ is an increasing function of $r$, Theorem A implies that if $f \in N$ then

$$
\log \frac{1}{1-r}-T\left(r, f^{\prime}\right) \longrightarrow \infty, \text { as } r \longrightarrow \mathrm{I} .
$$


Also, since the function $\mu$ of Theorem $\mathrm{B}$ is increasing, (iii) shows that (i) is equivalent to

$$
\log \frac{1}{1-r}-\mu(r) \uparrow \infty, \text { as } r \uparrow 1 \text {. }
$$

The author has recently obtained in [9] the analogues of Kennedy's results for analytic functions with finite Dirichlet integral in $U$.

The function $f$ constructed by Kennedy to prove Theorem $\mathrm{B}$ is given by a power series $\sum c_{k} z^{n_{k}}$ with Hadamard gaps such that $\sum\left|c_{k}\right|^{2}<\infty$. Such a function belongs to $H^{p}, 0<p<\infty$, and, even more, to VMOA. This follows from Paley's multiplier theorem [3,p. 104] and the duality of $H^{1}$ and BMOA $[7$, p. 270]. Hence (1.3) and (1.5) are sharp (in the sense of Theorem $B$ ) for VMOA functions.

The question as to whether or not there exists a function $f$ analytic and bounded in $U$ with $f^{\prime}$ satisfying the conclusion of Theorem $\mathrm{B}$ remains open. Kennedy pointed out in [14] that in dealing with this problem one could exclude functions $f(z)=\sum a_{k} z^{n_{k}}$ having Hadamard gaps. This is because if such a function is bounded in $U$ then $\sum\left|a_{k}\right|<\infty$ [20, vol. I p. 149 and 247] and so

$$
\int_{0}^{1} \exp T\left(r, f^{\prime}\right) d r<\infty
$$

a stronger inequality than (1.5). Clunie proved in [2] that there exists a function $f$ analytic and bounded in $U$ not satisfying (1.6).

A function $f$ analytic in $U$ is said to be a Bloch function if

$$
\|f\|_{B}=\sup _{|z|<1}\left(1-|z|^{2}\right)\left|f^{\prime}(z)\right|+|f(0)|<\infty
$$

The space of all Bloch functions will be denoted by $B$. Two important subspaces of $B$ are those denoted by $B_{0}$ and $B_{1}$. The space $B_{0}$ consists of those $f \in B$ such that $\left(1-|z|^{2}\right)\left|f^{\prime}(z)\right| \longrightarrow 0$, as $|z| \longrightarrow 1$. Alternatively, $B_{0}$ can be characterized as the closure of the polynomials in the Bloch norm [1, Th. 2.1].

The space $B_{1}$ consists of those $f \in B$ such that if $\left\{z_{n}\right\} \subset U$ and $\left|f\left(z_{n}\right)\right| \rightarrow \infty$ then $\left(1-\left|z_{n}\right|^{2}\right)\left|f^{\prime}\left(z_{n}\right)\right| \longrightarrow 0$. Clearisy, $B_{0} \subset B_{1}$.

If $f(z)=\sum_{n=0}^{\infty} a_{n} z^{n} \in B$ then $\sup \left|a_{n}\right|<\infty$ [1] while if $f \in B_{0}$ then $a_{n} \longrightarrow 0$. Actually, the weaker condition

$$
I_{2}\left(r, f^{\prime}\right)=o\left((1-r)^{-2}\right), \text { as } r \longrightarrow 1,
$$

is enough to conclude that $a_{n} \longrightarrow 0[16$, p. 693]. Here, for $g$ analytic in $U$

$$
I_{2}(r, g)=\frac{1}{2 \pi} \int_{-\pi}^{\pi}\left|g\left(r e^{i t}\right)\right|^{2} d t, \quad 0 \leq r<1 .
$$


The space $B_{1}$ was introduced in $[10$, p. 30] and $[1$, p. 36] where it was conjectured that if $f(z)=\sum_{n=0}^{\infty} a_{n} z^{n} \in B_{1}$ then $a_{n} \longrightarrow 0$. This was disproved by Fernández [4], [5]. Fernández gave in [4] examples of functions $f \in B_{1}$ not satisfying (1.8). If $D$ is a $B_{1}$-domain, i.e. if every function $g$ analytic in $U$ with $g(U) \subset D$ is in $B_{1}$, and $f$ is the universal covering map of $D$ then Hayman, Patterson and Pommerenke proved in [12] that $f$ satisfies (1.8) but Fernández proved in [5] that there exists a function $f$ analytic in $U$ whose range lies in a $B_{1}$-domain for which (1.8) is not true. The integral means and radial growth of $B_{1}$-functions were studied by the author in [8].

Important examples of Bloch functions are given by power series with Hadamard gaps, i.e. power series $f(z)=\sum_{n=0}^{\infty} a_{k} z^{n_{k}}$ analytic in $U$ with $n_{k+1}>\lambda n_{k}$ for some constant $\lambda>1$. For such an $f$ we have [1, p. 19] $f \in B$ if and only if $\sup \left|a_{k}\right|<\infty$ and [4], [16] $f \in B_{0} \Leftrightarrow f \in B_{1} \Leftrightarrow a_{k} \longrightarrow 0$.

It is well known that VMOA $\subset B_{0}$ and BMOA $\subset B$. The main object of this paper is studying whether or not (1.3) and (1.5), which are sharp for VMOA functions, remain true for functions in the spaces $B, B_{1}$, or $B_{0}$.

If $f \in B$ then

$$
T\left(r, f^{\prime}\right) \leq \log \frac{1}{1-r}+O(1)
$$

The first result in this paper asserts that this is essentially the best that we can say, showing that (1.5) and, hence, (1.3) need not be true for a Bloch function. However, we will prove that (1.5) holds for any $f \in B_{0}$ even though it may not satisfy (1.3).

Theorem 1. For each integer $q \geq 5$ let

$$
f_{q}(z)=\sum_{k=0}^{\infty} z^{q^{k}}, \quad|z|<1 .
$$

Then $f_{q}$ is a Bloch function and there exists a constant $C_{q}$ such that

$$
T\left(r, f^{\prime}\right) \geq \log \frac{1}{1-r}+C_{q}, \quad 0<r<1 .
$$

Theorem 2. (i) Let $f$ be a Bloch function satisfying

$$
I_{2}\left(r, f^{\prime}\right)=o\left((1-r)^{-2}\right) \text {, as } r \rightarrow 1 \text {. }
$$

Then

$$
\log \frac{1}{1-r}-T\left(r, f^{\prime}\right) \longrightarrow \infty, \text { as } r \longrightarrow \mathrm{I} .
$$

(ii) This result is best possible, even in $B_{0}$, in the following sense. Let $\Phi$ be a positive function in $[1, \infty)$ satisfying

$$
\lim _{x \rightarrow \infty} \Phi(x)=0 .
$$




$$
\Phi(2 x) \sim \Phi(x) \text {, as } x \longrightarrow \infty .
$$

Let

$$
\Psi(r)=\Phi\left(\frac{1}{1-r}\right), \quad 0 \leq r<1 .
$$

Then there exists $f \in B_{0}$ such that

$$
T\left(r, f^{\prime}\right) \geq \log \frac{1}{1-\tau}+\log \Psi(r)
$$

for all $r$ sufficiently close to 1 .

Let us notice that the function $\Psi$ of Theorem 2 can be taken to be

$$
\Psi(r)=\left(\log \frac{e}{1-r}\right)^{-1 / 2}, \quad 0 \leq r<\mathrm{I},
$$

and, hence, we obtain.

Corollary 1. There exists $f \in B_{0}$ such that

$$
T\left(r, f^{\prime}\right) \geq \log \frac{1}{1-r}-\frac{1}{2} \log \log \frac{e}{1-r}
$$

for all $r$ sufficiently close to 1 and, hence, satisfying

$$
\int_{0}^{1}(1-r) \exp \left(2 T\left(r, f^{\prime}\right)\right) d r=\infty .
$$

If $D$ is a $B_{1}$-domain and $f$ is the universal covering map of $D$ then [12] $f$ satisfies (1.8) and, hence, (1.5). It is known that (1.8) may not be true for a function in $B_{1}$ [4], [5]. We do not know whether or not (1.5) remains true for any function $f \in B_{1}$. We can prove the following result.

Theorem 3. Let $f \in B_{1}$. Then

$$
\liminf _{r \rightarrow 1}(1-r)^{2} I_{2}\left(r, f^{\prime}\right)=0
$$

and

$$
\limsup _{r \rightarrow 1}\left(\log \frac{1}{1-r}-T\left(r, f^{\prime}\right)\right)=\infty
$$

The early stages of this work benefited from conversations with A. Baernstein. He even told me that the conclusion of Theorem 1 should be true at least for sufficiently large values of $q$. It is a pleasure to express my gratitude. 


\section{Proof of the main results}

Even though the motivation of this work was studying the possibility of extending Kennedy's results to Bloch functions, some of our results are more general than stated and, in fact, could be stated without making any reference to Bloch functions.

For $f$ analytic in $U$ and $0 \leq r<1$, define

$$
\begin{aligned}
& I_{p}(r, f)=\frac{1}{2 \pi} \int_{-\pi}^{\pi}\left|f\left(r e^{i t}\right)\right|^{p} d t, \quad 0<p<\infty, \\
& M_{p}(r, f)=I_{p}(r, f)^{1 / p}, \quad 0<p<\infty, \\
& M_{\infty}(r, f)=\max _{|z|=r}|f(z)| .
\end{aligned}
$$

For $s>0$ and $0<p \leq \infty$, let $X^{s, p}$ denote the space of those functions $f$ analytic in $U$ for which

$$
M_{p}(r, f)=O\left((1-r)^{-s}\right), \text { as } r \longrightarrow 1,
$$

and let $X_{0}^{s, p}$ denote the space of those functions $f$ analytic in $U$ for which

$$
M_{p}(r, f)=o\left((1-r)^{-s}\right), \text { as } r \longrightarrow 1 .
$$

Since $M_{p}(r, f)$ is an increasing function of $p$, we have

$$
X^{s, p} \subset X^{s, p^{\prime}} \text { and } X_{0}^{s, p} \subset X_{0}^{s, p^{\prime}}, \quad 0<p^{\prime}<p \leq \infty .
$$

If $p \geq 1$ and $f(z)=\sum_{n=0}^{\infty} a_{n} z^{n} \in X^{s_{+} p}$ (respectively $X_{0}^{s, p}$ ) then an application of Cauchy's formula easily gives $a_{n}=O\left(n^{s}\right)$ (respectively $a_{n}=o\left(n^{s}\right)$ ). On the other hand, an argument similar to that used in [16, Example 1, p. 694] proves that if $f(z) \sum_{k=0}^{\infty} a_{k} z^{n_{k}}$ is analytic in $U$ and has Hadamard gaps then

$$
f \in X^{S, \infty} \Leftrightarrow a_{k}=O\left(n_{k}^{s}\right), \text { as } k \longrightarrow \infty,
$$

and

$$
f \in X_{0}^{S, \infty} \Leftrightarrow a_{k}=o\left(n_{k}^{s}\right), \text { as } k \longrightarrow \infty
$$

Let us notice that, clearly

$$
f \in B \Leftrightarrow f^{\prime} \in X^{1, \infty} \text { and } f \in B_{0} \Leftrightarrow f^{\prime} \in X_{0}^{1, \infty} .
$$

Hence theorems 1 and 2 will be corollaries of the more general results that wc will prove for the spaces $X^{s, p}$ and $X_{0}^{s, p}$. 
If $p<p^{\prime}$ and $f \in X^{s, p}$ (respectively $X_{0}^{s, p}$ ) then a result of Hardy and Littlewood (see $[3, T h .5 .9]$ ) shows that $f \in X^{s^{\prime}, p^{\prime}}$ (respectively $X_{0}^{s^{\prime}, p^{\prime}}$ ) where

$$
s^{\prime}=s+\frac{1}{p}-\frac{1}{p^{\prime}}
$$

The exponent $s^{\prime}$ is best possible.

Using this result and arguing as in [3, Th. 6.4] we can deduce that if $0<p<I$ and $f(z)=\sum_{n=0}^{\infty} a_{n} z^{n} \in X^{s, p}$ then

$$
a_{n}=0\left(n^{s-1+1 / p}\right) .
$$

The function $f(z)=(1-z)^{-(s+1 / p)}$ for which $a_{n} \sim \Gamma\left(s+\frac{1}{p}\right) n^{s-1+1 / p}$ shows that this estimate is sharp.

Now, if $p^{\prime}<p$ and $f \in X^{s, p}$ then it is easy to sec that the trivial result $f \in X^{s, p^{\prime}}$ is the best that we can say in general. In fact, there exists $f \in X^{s, \infty}$ such that for every $p \in(0, \infty]$ there exists a constant $B_{p, s}>0$ such that

$$
M_{p}(r, f) \geq B_{p_{r} s}(1-r)^{-s}, \quad \frac{1}{2} \leq r<1, \quad 0<p \leq \infty .
$$

Indeed, let $q \geq 2$ be an integer and

$$
f(z)=\sum_{k=0}^{\infty} q^{k s} z^{q^{k}}, \quad|z|<1 .
$$

Then, since $f$ has Hadamard gaps, (2.2) shows that $f \in X^{s, \infty}$. Now, it is a simple exercise to show that there exists a constant $\beta_{s}=\beta_{s, q}>0$ such that

$$
M_{2}(r, f) \geq \beta_{s}(1-r)^{-s}, \quad \frac{1}{2} \leq r<1 .
$$

This implies (2.4) for $2 \leq p \leq \infty$ with $B_{p, s}=\beta_{s}$. Using Theorem 8.20 of $[20$, vol. I, p. 215] we deduce that for each $p \in(0,2)$ there exists a constant $A_{p}=A_{p, q}>0$ such that

$$
M_{p}(r, f) \geq A_{p} M_{2}(r, f), \quad 0 \leq r<1, \quad 0<p<2,
$$

which, with (2.5) implies (2.4) for $0<p<2$ with

$$
B_{p, s}=\beta_{s} A_{p}
$$

Since

$$
\log M_{p}(r, f) \downarrow \frac{1}{2 \pi} \int_{-\pi}^{\pi} \log \left|f\left(r e^{i t}\right)\right| d t, \text { as } p \downarrow 0
$$


(2.4) shows that, for $\frac{1}{2} \leq r<1$,

$$
T(r, f) \geq \frac{1}{2 \pi} \int_{-\pi}^{\pi} \log \left|f\left(r e^{i t}\right)\right| d t \geq s \log \frac{1}{1-\tau}+\gamma_{s}
$$

wherc

$$
\gamma_{s}=\liminf _{p \longrightarrow 0} \log B_{p, s} .
$$

An examination of the proof of Theorem 8.20 in [20, vol. I, p. 215] shows that the constant $A_{p}$ given there is of the form

$$
A_{p}=\delta_{q}^{(p-2) / p}
$$

for some $\delta_{q}>1$. This and (2.7) shows that $\gamma_{s}=-\infty$ and hence (2.9) gives no information at all.

However, we will prove in Theorem 4 that there exists $f \in X^{s, \infty}$ satisfying (2.9) with a constant $C_{s}$ in the place of $\gamma_{s}$ and, also, satisfying (2.4) with a constant $B_{s}>0$ independent of $p$ in the place of $B_{p, s}$.

Theorem 4. (i) Let $s>0,0<p \leq \infty$ and $f \in X^{s, p}$, then

$$
f \in X^{s, p^{\prime}}, \quad 0<p^{\prime}<p
$$

and

$$
T(r, f) \leq s \log \frac{1}{1-r}+O(1)
$$

(ii) This result is best possible in the following sense. There exists $f \in X^{s, \infty}$ and two constants $C_{s} \in \mathbb{P}$ and $B_{s}>0$ such that

$$
M_{p}(r, f) \geq B_{s}(1-r)^{-s}, \quad 0 \leq r<1, \quad 0<p \leq \infty,
$$

and

$$
T(r, f) \geq \frac{1}{2 \pi} \int_{-\pi}^{\pi} \log \left|f\left(r e^{i t}\right)\right| d t \geq s \log \frac{1}{1-r}+C_{s}, \quad 0 \leq r<1 .
$$

For $s=1$, the conclusion of (ii) holds with $f=f_{q}^{\prime}$ for any integer $q \geq 5$.

Theorem 5 gives the analogous results for the spaces $X_{0}^{s, p}$. 
Theorem 5. (i) Let $s>0,0<p \leq \infty$ and $f \in X_{0}^{s, p}$. Then

$$
f \in X_{0}^{s, p^{\prime}}, \quad 0<p^{\prime} \leq p
$$

and

$$
s \log \frac{1}{1-r}-T(r, f) \longrightarrow \infty, \text { as } r \longrightarrow 1 .
$$

(ii) This result is best possible in the following sense. Let $\Phi$ be a positive function in $[1, \infty)$ satisfying (1.12) and (1.13) and let

$$
\Psi(r)=\Phi\left(\frac{1}{1-r}\right), \quad 0 \leq r<1 .
$$

Then there exists $f \in X_{0}^{s, \infty}$ such that

$$
\frac{(1-r)^{s}}{\Psi(r)} M_{p}(r, f) \longrightarrow \infty \text {, as } r \longrightarrow 1, \text { for every } p \in(0, \infty]
$$

and

$$
T(r, f) \geq \frac{1}{2 \pi} \int_{-\pi}^{\pi} \log \left|f\left(r e^{i t}\right)\right| d t \geq s \log \frac{1}{1-r}+\log \Psi(r)
$$

for all $r$ sufficiently close to 1 .

Proof of Theorems 4(i) and 5(i): We have already proved (2.10) and (2.15). Also, (2.11) and (2.16) are obvious for $p=\infty$.

Now, let $f$ be a function analytic in $U$ and $0<p<\infty$. Using the arithmeticgeometric inequality, we obtain

$$
\begin{aligned}
T(r, f)=\frac{1}{2 \pi} \int_{-\pi}^{\pi} & \log ^{+}\left|f\left(r e^{i t}\right)\right| d t \leq \frac{1}{p} \frac{1}{2 \pi} \int_{-\pi}^{\pi} \log \left(\left|f\left(r e^{i t}\right)\right|^{p}+1\right) d t \\
& \leq \frac{1}{p} \log \left(\frac{1}{2 \pi} \int_{-\pi}^{\pi}\left(\left|f\left(r e^{i t}\right)\right|^{p}+1\right) d t=\frac{1}{p} \log \left(I_{p}(r, f)+1\right) .\right.
\end{aligned}
$$

Hence

$$
s \log \frac{1}{1-r}-T(r, f) \geq \frac{1}{p} \log \frac{1}{(1-r)^{s p}\left(I_{p}(\tau, f)+1\right)} .
$$

Then it is clear that $(2.11)$ (respectively $(2.16)$ ) holds if $f \in X^{s, p}$ (respectively if $f \in X_{0}^{s, p}$ ).

Proof of Theorem 4(ii): Let $s>0$. Let $q \geq 2$ be an integer to be determined later and

$$
f(z)=\sum_{k=0}^{\infty} q^{k s} z^{q^{k}-1}, \quad|z|<1 .
$$


Then, since $f$ is given by a power series with Hadamard gaps, (2.2) shows that $f \in X^{s, \infty}$. Let

$$
r_{n}=1-q^{-n}, \quad n=1,2,3, \ldots
$$

Then, for $|z|=r_{n}$

$|f(z)|>|z f(z)|=\left|\sum_{k=0}^{\infty} q^{k s} z^{q^{k}}\right| \geq q^{n s} r_{n}^{q^{n}}-\sum_{k=0}^{n-1} q^{k s}-\sum_{k=n+1}^{\infty} q^{k s} r_{n}^{q^{k}}=I-I I-I I I$.

Let $\eta \in(0,1)$ to be determined later. Using the elementary fact

$$
\left(1-\frac{1}{j}\right)^{j} \longrightarrow e^{-1}, \text { as } j \longrightarrow \infty
$$

we deduce that there exists $N$ such that

$$
I=q^{n s}\left(1-q^{-n}\right)^{q^{n}} \geq(1-\eta) e^{-1} q^{n s}, \quad n \geq N .
$$

Now,

$$
I I=\sum_{k=0}^{n-1} q^{k s}=\frac{q^{n s}-1}{q^{s}-1}<\frac{q^{n s}}{q^{s}-1}
$$

In order to obtain an upper bound for $I I I$ we will use the following lemma which will be needed several times in this paper.

Lemma 1. Let $q \geq 2$ be an integer and let $s>0$. Let $\left\{a_{k}\right\}$ be a nonincreasing sequence of positive numbers. Then, for any integer $m>s$, we have

$$
\sum_{k=n+1}^{\infty} a_{k} q^{k s}\left(1-q^{-n}\right)^{q^{k}} \leq\left(m e^{-1}\right)^{m} a_{n} \frac{1}{q^{m-s}-1} q^{n s}, \quad n=1,2,3, \ldots
$$

Proof of lemma 1: We will use the following elementary inequality (2.21)

$$
(1-x)^{n} \leq\left(m e^{-1}\right)^{m}(n x)^{-m},(0<x<1, n=1,2,3, \ldots, m=1,2,3, \ldots) .
$$

Let $m>s$ be an integer; then, since $\left\{a_{k}\right\}$ is non-increasing, (2.21) implies

$$
\begin{aligned}
\sum_{k=n+1}^{\infty} a_{k} q^{k s}\left(1-q^{-n}\right)^{q^{k}} \leq\left(m e^{-1}\right)^{m} a_{n} \sum_{k=n+1}^{\infty} q^{k s} q^{-m k} q^{n m} \\
=\left(m e^{-1}\right)^{m} a_{n} q^{n m} \sum_{k=n+1}^{\infty} q^{k(s-m)}=\left(m e^{-1}\right)^{m} a_{n} \frac{q^{n s}}{q^{m-s}-1}
\end{aligned}
$$


Back to the proof of Theorem 4, take an integer $m>s$. Then, using Lemma 1 with $a_{k}=1$ for all $k$, we obtain

$$
I I \leq\left(m e^{-1}\right)^{m} \frac{1}{q^{m-s}-1} q^{n s} .
$$

Then (2.17), (2.19), (2.20) and (2.22) show that

$$
|f(z)| \geq\left(M_{s, m, q}-\eta e^{-1}\right) q^{n s}, \quad|z|=r_{n}, \quad n \geq N,
$$

where,

$$
M_{s, m, q}=e^{-1}-\frac{1}{q^{s}-1}-\left(m e^{-1}\right)^{m} \frac{1}{q^{m-s}-1} .
$$

Now, take $q$ so large that $M_{s, m, q}>0$ and then take $\eta=\eta_{q}>0$ so small that

$$
T_{s}=M_{s, m, q}-\eta e^{-1}>0 .
$$

Then

$$
|f(z)| \geq T_{s} q^{n s}, \quad|z|=r_{n}, \quad n \geq N .
$$

Then, for $n \geq N$,

$$
\frac{1}{2 \pi} \int_{-\pi}^{\pi} \log \left|f\left(T_{n} e^{i t}\right)\right| d t \geq \log q^{n s}+\log T_{s}=s \log \frac{1}{1-r_{n}}+\log T_{s} .
$$

Now, since $\frac{1}{2 \pi} \int_{-\pi}^{\pi} \log \left|f\left(r e^{i t}\right)\right| d t$ is an increasing function of $r$, it follows that, for $n \geq N$ and $r_{n} \leq r<r_{n+1}$,

$$
\begin{aligned}
T(r, f) \geq \frac{1}{2 \pi} \int_{-\pi}^{\pi} \log \left|f\left(r e^{i t}\right)\right| d t & \geq \frac{1}{2 \pi} \int_{-\pi}^{\pi} \log \left|f\left(r_{n} e^{i t}\right)\right| d t \\
\geq s \log \frac{1}{1-r_{n}}+\log T_{s}=s \log \frac{1}{1-r_{n+1}}-s \log q+\log T_{s} & >s \log \frac{1}{1-r}+\log \left(T_{s} / q^{s}\right) .
\end{aligned}
$$

Consequently, we have

$$
T(r, f) \geq \frac{1}{2 \pi} \int_{-\pi}^{\pi} \log \left|f\left(r e^{i t}\right)\right| d t>s \log \frac{1}{1-r}+\log \left(T_{s} / q^{s}\right), \quad r_{N} \leq r<1,
$$

which clearly implies (2.14) with

$$
C_{s}=\min \left\{\inf _{0 \leq r \leq r_{N}}\left(\frac{1}{2 \pi} \int_{-\pi}^{\pi} \log \left|f\left(r e^{i t}\right)\right| d t-s \log \frac{1}{1-r}\right), \log \left(T_{s} / q^{s}\right)\right\} .
$$


Now (2.8) and (2.14) prove (2.13) with

$$
B_{s}=\exp C_{s}
$$

Let us notice that in the above argument given $s>0$ we can take $m$ to be any integer greater than $s$ and that different values of $m$ would lead to different values of $q$ for which $M_{s, m, q}>0$. It turns out that for $s=1$ the choice of $m$ which minimizes $q$ is $m=3$. If we set

$$
\lambda_{q}=M_{1,3, q}
$$

we have $\lambda_{q}>0$ for $q \geq 5$. In fact, we have

$$
\lambda_{q} \geq \lambda_{5} \simeq 0.061, \quad q \geq 5 .
$$

Consequently, we obtain that for each integer $q \geq 5$ there exists a constant $C_{q}$ such that

$$
T\left(r, f_{q}^{\prime}\right) \geq \log \frac{1}{1-r}+C_{q}, \quad 0 \leq r<1 .
$$

This finishes the proof of theorems 4 and 1 .

Proof of Theorem 5(ii): First let us notice that we may assume without loss of generality that $\Phi$ satisfies also the following two conditions.

$$
\Phi \text { is decreasing, }
$$

Indeed let $\Phi$ satisfy the conditions of Theorem 5 . Let

$$
\Phi_{1}(x)=\max \left(\Phi(x),(\log 2 x)^{-1}\right)
$$

and let $\Phi_{2}$ denote the least decreasing majorant of $\Phi_{1}$,

$$
\Phi_{2}(x)=\sup _{t \geq x} \Phi_{1}(t)
$$

Then it is clear that $\Phi_{2} \geq \Phi$ and it is easy to see that (1.12), (1.13), (2.23) and (2.24) hold with $\Phi_{2}$ in the place of $\Phi$.

Hence we will assume that $\Phi$ satisfies (2.23) and (2.24) in addition to the conditions of Theorem 5 .

Let $q \geq 2$ be an integer with $q^{9}>2$ to be dctcrmined later and set

$$
g(z)=\sum_{k=1}^{\infty} \Phi\left(q^{k}\right) q^{k s} z^{q^{k}}, \quad|z|<\mathfrak{I} .
$$


Then, since $g$ is given by a power series with Hadamard gaps, (1.12) and (2.3) show that $g \in X_{0}^{s, \infty}$.

Let

$$
r_{n}=1-q^{-n}, \quad n=1,2,3, \ldots
$$

Then, for $|z|=r_{n}$,

$$
\begin{aligned}
|g(z)| & =\left|\sum_{k=1}^{\infty} \Phi\left(q^{k}\right) q^{k s} z^{q^{k}}\right| \geq \Phi\left(q^{n}\right) q^{n s}\left(1-q^{-n}\right)^{q^{n}} \\
& -\sum_{k=1}^{n-1} \Phi\left(q^{k}\right) q^{k s}-\sum_{k=n+1}^{\infty} \Phi\left(q^{k}\right) q^{k s}\left(1-q^{-n}\right)^{q^{k}}=I-I I-I I
\end{aligned}
$$

Using (2.18), we see that there exists $N$ such that

$$
I=\Phi\left(q^{n}\right) q^{n s}\left(1-q^{-n}\right) q^{n} \geq \frac{1}{2} e^{-1} \Phi\left(q^{n}\right) q^{n s}, \quad n \geq N
$$

Now, (1.13) and (2.23) imply

$$
\Phi(q x) \sim \Phi(x) \text {, as } x \longrightarrow \infty,
$$

and hence there exists $N_{1}>N$ such that

$$
\Phi\left(q^{k}\right)<2 \Phi\left(q^{k+1}\right), \quad k \geq N_{1} .
$$

Set

$$
C=\sum_{k=1}^{N_{1}} \Phi\left(q^{k}\right) q^{k s}
$$

Then (2.28) shows that, for $n>N_{1}+1$,

$$
\begin{gathered}
I I=\sum_{k=1}^{n-1} \Phi\left(q^{k}\right) q^{k s}=C+\sum_{k=N_{1}+1}^{n-1} \Phi\left(q^{k}\right) q^{k s} \leq \\
C+\sum_{k=N_{1}+1}^{n-1} 2^{n-k} \Phi\left(q^{n}\right) q^{k s}<C+2^{n} \Phi\left(q^{n}\right) \sum_{k=1}^{n-1} 2^{-k} q^{k s}<C+\frac{2}{q^{s}-2} \Phi\left(q^{n}\right) q^{n s} .
\end{gathered}
$$

Then (2.24) shows that there exists $N_{2}>N_{1}$ such that

$$
I I<\frac{3}{q^{s}-2} \Phi\left(q^{n}\right) q^{n s}, \quad n \geq N_{2} .
$$

Finally, fix an integer $m>s$. Then Lernma 1 shows that

$$
I I \leq\left(m e^{-1}\right)^{m} \frac{1}{q^{m-s}-1} \Phi\left(q^{n}\right) q^{n s} .
$$


Then if we take $q$ so large that

$$
\frac{3}{q^{s}-2}<\frac{1}{8 e} \text { and }\left(m e^{-1}\right)^{m} \frac{1}{q^{m-s}-1}<\frac{1}{8 e},
$$

$(2.25),(2.26),(2.29)$ and $(2.30)$ show that

$$
|g(z)| \geq(4 e)^{-1} \Phi\left(q^{n}\right) q^{n s}, \quad|z|=r_{n}, \quad n \geq N_{2} .
$$

Notice that (2.27) and the definition of $\Psi$ imply that

$$
\log \left(\Psi\left(r_{n}\right) / \Psi\left(r_{n+1}\right)\right) \longrightarrow 0, \text { as } n \longrightarrow \infty,
$$

which, together with (2.23) shows that there exists $N_{3}>N_{2}$ and a constant $A$ such that

$$
\log \Psi\left(r_{n}\right) \geq \log \Psi(r)+A, \quad T_{n} \leq r \leq r_{n+1}, \quad n \geq N_{3} .
$$

Let $\alpha=4 e q^{s} e^{-A}$ and $f(z)=\alpha g(z)$. Then $f \in X_{0}^{s, \infty}$. Since $\frac{1}{2 \pi} \int_{-\pi}^{\pi} \log \left|f\left(r e^{i t}\right)\right| d t$ is an increasing function of $r,(2.31),(2.32)$ and the definitions of $\Psi, r_{n}, N_{2}, N_{3}$ and $\alpha$ show that, for $n \geq N_{3}$ and $r_{n} \leq r<r_{n+1}$,

$$
\begin{aligned}
& T(r, f) \geq \frac{1}{2 \pi} \int_{-\pi}^{\pi} \log \left|f\left(r e^{i t}\right)\right| d t \geq \frac{1}{2 \pi} \int_{-\pi}^{\pi} \log \left|f\left(r_{n} e^{i t}\right)\right| d t \\
& \geq \log \alpha+s \log q^{n}+\log \Phi\left(q^{n}\right)+\log \frac{1}{4 e} \\
& =s \log q^{n+1}+\log \Phi\left(q^{n}\right)+\log \frac{\alpha}{4 e q^{s}} \\
& =s \log \frac{1}{1-r_{n+1}}+\log \Psi\left(r_{n}\right)+\log \frac{\alpha}{4 e q^{s}} \\
& >s \log \frac{1}{1-r}+\log \Psi(r)+A+\log \frac{\alpha}{4 e q^{s}} \\
& =s \log \frac{1}{1-T}+\log \Psi(r)+\log \frac{\alpha e^{A}}{4 e q^{s}}=s \log \frac{1}{1-r}+\log \Psi(r) .
\end{aligned}
$$

This proves (2.18) for $r_{N_{3}} \leq r<1$.

Now, (2.18) and (2.8) easily imply

$$
M_{p}(r, f) \geq \Psi(r)(1-r)^{-s}, \quad 0<p \leq \infty,
$$

for all $r$ sufficiently close to 1 .

In order to show that there exists $f \in X_{0}^{s, \infty}$ satisfying (2.17), let us notice that if $\Phi$ is the function given in Theorem 5 then $\Phi^{1 / 2}$ satisfies also the conditions (1.12) and (1.13). Hence, if we apply the above argument with $\Phi^{1 / 2}$ in the place of $\Phi$, we deduce that there exists $f \in X_{0}^{s, \infty}$ such that

$$
M_{p}(r, f) \geq \Psi(r)^{1 / 2}(1-r)^{-s}, \quad 0<p \leq \infty,
$$


for all $r$ sufficiently to 1 . Since $\Psi(r) \longrightarrow 0$, as $r \longrightarrow 1$, this implies (2.17).

For the proof of Theorem 3 we need to introduce the Ahlfors-Shimizu characteristic. For $f$ analytic in $U$, define

$$
S(r, f)=\frac{I}{\pi} \iint_{|z|<r} \frac{\left|f^{\prime}(z)\right|^{2}}{\left(1+|f(z)|^{2}\right)^{2}} d x d y, \quad 0 \leq r<1 .
$$

Then, the Ahlfors-Shimizu characteristic $T_{0}(r, f)$ is defined by

$$
T_{0}(r, f)=\int_{0}^{r} \frac{S(t, f)}{t} d t, \quad 0 \leq r<1 .
$$

Then $[11$, p. 13]

$$
\left|T(r, f)-T_{0}(r, f)-\log ^{+}\right| f(0)|| \leq \frac{1}{2} \log 2, \quad 0 \leq r<1 .
$$

We have

Lemma 2. Let $f$ be a Bloch function. Then

$$
\liminf _{r \longrightarrow 1}(1-r) \log \frac{1}{1-r} S(r, f) \leq \frac{1}{2}
$$

Proof: Let $f \in B$. Anderson, Clunie and Pommerenke proved in [1, Th. 3.1] that

$$
\limsup _{r \rightarrow 1} \frac{T(r, f)}{\log \log \frac{1}{1-r}} \leq \frac{1}{2}
$$

Then (2.35) shows that

$$
\limsup _{r \rightarrow 1} \frac{T_{0}(r, f)}{\log \log \frac{1}{1-r}} \leq \frac{1}{2}
$$

and hence

$$
\liminf _{r \rightarrow 1} \frac{\frac{d}{d r} T_{0}(r, f)}{\frac{d}{d r} \log \log \frac{l}{1-r}} \leq \frac{1}{2}
$$

which is equivalent to (2.36).

Proof of Theorem 3: Let $f \in B_{1}$. Then (2.36) implies

$$
\liminf _{r \rightarrow 1}(1-r) S(r, f)=0
$$

Let $\eta>0$. Since $f \in B_{1}$, there exists $M>0$ such that

$$
(1-|z|)\left|f^{\prime}(z)\right|<\eta \text { if }|f(z)|>M
$$


Let

$$
\begin{aligned}
& F_{r}=\{z:|z|<r \text { and }|f(z)| \leq M\} \\
& G_{r}=\{z:|z|<r \text { and }|f(z)|>M\}
\end{aligned}
$$

Then (2.38) implies

$$
\begin{aligned}
\iint_{|z|<r}\left|f^{\prime}(z)\right|^{2} d x d y=\iint_{F_{r}}\left|f^{\prime}(z)\right|^{2} d x d y & +\iint_{G_{r}}\left|f^{\prime}(z)\right|^{2} d x d y \\
\leq\left(1+M^{2}\right)^{2} \iint_{|z|<r} \frac{\left|f^{\prime}(z)\right|^{2}}{\left(1+|f(z)|^{2}\right)^{2}} d x d y & +\iint_{|z|<r} \frac{\eta^{2}}{(1-|z|)^{2}} d x d y \\
& \leq\left(1+M^{2}\right)^{2} \pi S(r, f)+\frac{2 \pi \eta^{2}}{1-r}
\end{aligned}
$$

Hence, using (2.37), we obtain

$$
\liminf _{r \longrightarrow 1}(1-r) \iint_{|z|<r}\left|f^{\prime}(z)\right|^{2} d x d y \leq 2 \pi \eta^{2}
$$

Since $\eta>0$ is arbitrary, we have

$$
\liminf _{r \longrightarrow 1}(1-r) \iint_{|z|<r}\left|f^{\prime}(z)\right|^{2} d x d y=0
$$

which implies

$$
\liminf _{r \rightarrow 1} \frac{\frac{d}{d r} \iint_{|z|<r}\left|f^{\prime}(z)\right|^{2} d x d y}{\frac{d}{d r} \frac{1}{1-r}}=0
$$

and this is equivalent to (1.15).

Now, an argument similar to that used in the proof of Theorem 5(i) shows that (1.15) implies (1.16). This finishes the proof of Theorem 3.

\section{Some further results and final remarks}

a) The results that we have proved are comparison results between $M_{p}(r, f)$ with $M_{p^{\prime}}(r, f)$ and $T(r, f)$ for $f$ in some of the spaces $X^{s, p}$ or $X_{0}^{s, p}$. It is well known (see e.g. [3, Th. 5.10]) that there exist functions $f$ analytic in $U$ with $M_{\infty}(r, f)$ growing to infinity arbitrarily slowly which are not of bounded characteristic. This leads one to ask the following question:

Let $\mu(r)$ be a positive increasing function on $0 \leq r<1$ with $\mu(0)=1$ and $\mu(r) \longrightarrow \infty$, as $r \longrightarrow 1$, and let $f$ be a function analytic in $U$ satisfying $M_{p}(r, f)=0(\mu(r))$, as $r \longrightarrow 1$. What can be said about the growth of $M_{p^{\prime}}(r, f)$ and $T(r, f)$ ? In particular, it seems natural to ask whether or not the analogue 
of Theorem 4(ii) is true in this setting, i.e. does there exist a function $f$ analytic in $U$ with

$$
M_{\infty}(r, f)=0(\mu(r)), \text { as } r \longrightarrow 1,
$$

and a constant $C$ such that

$$
T(r, f) \geq \log \mu(\tau)+C, \quad 0 \leq r<1 ?
$$

We do not know the answer to this question. However, we do believe that the methods of this paper are not enough to construct such an $f$.

b) First of all let us remark that some of the results that we are going to state below (Theorem 6, Corollary 2, and Theorem 7) could be stated in the general framework of the spaces $X^{s, p}$ and $X_{0}^{s, p}$. However, for the sake of simplicity, we will state them in the setting of Bloch functions.

It seems natural to conjecture that the conclusion of Theorem 1 remains true for $q=2,3$, and 4 . However, our argument does not prove this since, with the notation used in the proof of Theorem I, we have $\lambda_{4}<0$.

A more general question would be characterizing those Bloch functions given by a power series with Hadamard gaps for which (1.5) or at least (1.16) is true. The following theorem gives a partial answer to this question.

Theorem 6. Let $f$ be a Bloch function given by a power series

$$
f(z)=\sum_{j=1}^{\infty} a_{j} z^{n_{j}}, \text { with } \frac{n_{j}+1}{n_{j}} \longrightarrow \infty, \text { as } j \longrightarrow \infty .
$$

Then

$$
\limsup _{r \rightarrow 1}\left(\log \frac{1}{1-r}-T\left(r, f^{\prime}\right)\right)=\infty
$$

Furthermore, if $\limsup _{j \rightarrow \infty}\left|a_{j}\right|>0$ then

$$
\liminf _{r \rightarrow 1}\left(\log \frac{1}{1-r}-T\left(r, f^{\prime}\right)\right)<\infty
$$

Using Theorem 2, we obtain as an easy consequence of Theorem 6 the following result.

Corollary 2. Let $f$ be a Bloch function given by a power series

$$
f(z)=\sum_{j=1}^{\infty} a_{j} z^{n_{j}} \text { with } \frac{n_{j+1}}{n_{j}} \rightarrow \infty, \text { as } j \longrightarrow \infty .
$$

Then $f \in B_{0}$ if and only if

$$
\lim _{r \rightarrow 1}\left(\log \frac{1}{1-r}-T\left(r, f^{\prime}\right)\right)=\infty
$$

The proof of Theorem 6 depends on the following two elementary lemmas whose proofs will be omitted. 
Lemma 3. [14, p. 339] Let $\left\{s_{k}\right\}$ be a sequence of positive numbers such that $s_{k+1} / s_{k} \longrightarrow \infty$ as $k \longrightarrow \infty$. Then, as $k \longrightarrow \infty$,

$$
\sum_{j=1}^{k-1} s_{j}=o\left(t_{k}\right) \text { and } \sum_{j=k+1}^{\infty} s_{j}^{-1}=o\left(s_{k}^{-1}\right)
$$

Lemma 4. Let $\left\{s_{k}\right\}$ and $\left\{t_{k}\right\}$ be two sequences of positive numbers such that

$$
s_{k+1} / t_{k} \longrightarrow \infty \text { and } t_{k} / s_{k} \longrightarrow \infty \text {, as } k \longrightarrow \infty
$$

Then, as $k \longrightarrow \infty$,

$$
\sum_{j=1}^{k} s_{j}=o\left(t_{k}\right) \text { and } \sum_{j=k+1}^{\infty} s_{j}^{-1}=o\left(t_{k}^{-1}\right)
$$

Proof of Theorem 6: Let $f$ be a Bloch function given by (3.1). Since $f \in B$, there exists $K>0$ such that

$$
\left|a_{j}\right| \leq K, \quad j=1,2,3, \ldots
$$

Let $\left\{m_{j}\right\}$ be an increasing sequence of positive numbers such that

$$
\frac{m_{j}}{n_{j}} \longrightarrow \infty \text { and } \frac{n_{j+1}}{m_{j}} \longrightarrow \infty \text {, as } j \longrightarrow \infty \text {. }
$$

For example, we can take $m_{j}=\left(n_{j} n_{j+1}\right)^{1 / 2}$.

Let $|z|=1-m_{k}^{-1}$. Then

$$
\left|z f^{\prime}(z)\right|=\left|\sum_{j=1}^{\infty} n_{j} z^{n_{j}}\right| \leq K \sum_{j=1}^{k} n_{j}+K \sum_{j=k+1}^{\infty} n_{j}\left(1-m_{k}^{-1}\right)^{n_{j}} .
$$

Using (3.3) and Lemma 4, we obtain

$$
\sum_{j=1}^{k} n_{j}=o\left(m_{k}\right), \text { as } k \longrightarrow \infty
$$

Now, (2.21) with $m=3,(3.3)$ and Lemma 4 imply

$$
\begin{aligned}
\sum_{j=k+1}^{\infty} n_{j}\left(1-m_{k}^{-1}\right)^{n_{j}} \leq & \left(3 e^{-1}\right)^{3} m_{k}^{3} \sum_{j=k+1}^{\infty} n_{j}^{-2} \\
& =\left(3 e^{-1}\right)^{3} m_{k}^{3} o\left(m_{k}^{-2}\right)=o\left(m_{k}\right), \text { as } k \longrightarrow \infty
\end{aligned}
$$


Then (3.4), (3.5) and (3.6) show that

$$
\sup _{|z|=1-m_{k}^{-1}}\left|z f^{\prime}(z)\right|=o\left(m_{k}\right), \text { as } k \longrightarrow \infty,
$$

and hence

$$
\log m_{k}-T\left(1-m_{k}^{-1}, f^{\prime}\right) \longrightarrow \infty, \text { as } k \longrightarrow \infty .
$$

This implies that

$$
\limsup _{r \rightarrow 1}\left(\log \frac{1}{1-r}-T\left(r, f^{\prime}\right)\right)=\infty
$$

Assume now that $f$ is given by (3.1), satisfies (3.2) and $\limsup _{k \rightarrow \infty}\left|a_{k}\right|>0$. Then there exists $M>0$ such that the set

$$
T=\left\{k:\left|a_{k}\right|>M\right\}
$$

is infinite. Take $k \in T$ and let $r_{k}=1-n_{k}^{-1}$. Then, for $|z|=r_{k}$

$$
\begin{aligned}
& \left|f^{\prime}(z)\right|>\left|z f^{\prime}(z)\right| \\
& \quad \geq M n_{k}\left(1-n_{k}^{-1}\right)^{n_{k}}-K \sum_{j=1}^{k-1} n_{j}-K \sum_{j=k+1}^{\infty} n_{j}\left(1-n_{k}^{-1}\right)^{n_{j}}=I-I I-I I I .
\end{aligned}
$$

Using (2.18), we see that there exists a constant $C>0$ such that

$$
I \geq C n_{k} .
$$

Lemma 3 implies that

$$
I I=o\left(n_{k}\right), \text { as } k \longrightarrow \infty .
$$

Finally, (2.21) and Lemma 3 show that

$$
I I I=o\left(n_{k}\right), \text { as } k \longrightarrow \infty .
$$

Consequently, we obtain that

$$
\inf _{|z|=r_{k}}\left|f^{\prime}(z)\right| \geq C n_{k}-o\left(n_{k}\right), \text { as } k \longrightarrow \infty(k \in T),
$$

and, hence

$$
T\left(r_{k}, f^{\prime}\right) \geq \log \left(n_{k}-o\left(n_{k}\right)\right)+O(1), \text { as } k \longrightarrow \infty(k \in T) .
$$

This easily implies that

$$
\liminf _{r \rightarrow 1}\left(\log \frac{1}{1-r}-T\left(r, f^{\prime}\right)\right)<\infty
$$

finishing the proof of Theorem 6 .

c) So far we have proved in Theorem 2 that if a Bloch function $f$ satisfies (1.8) then it satisfies (1.5). Furthermore, the functions $f$ considered in theorems 3 and 6 satisfy not only (1.16) but also (1.15). These facts might lead one to ask whether or not the converse of Theorem 2(i) is true. Theorem 7 shows that the answer to this question is negative in a very strong sense. 
Theorem 7, Let $0<H<1$. Then there exists a Bloch function $f$ such that

$$
\liminf _{r \longrightarrow 1}(1-r)^{2} I_{2}\left(r, f^{\prime}\right)>0
$$

and

$$
\lim _{r \rightarrow 1} \frac{T\left(r, f^{\prime}\right)}{\log \frac{1}{1-r}}=H
$$

Proof: We will use the following result due to Specht. [18, Th. III] (see also [13, Lem. I and 2]) on the conformal mapping of certain nearly circular regions.

There exists a simply connected domain $D$ in the plane with

$$
U \cup\left\{e^{i t}: \pi H<|t| \leq \pi\right\} \subset D
$$

and such that if $\omega$ denotes the conformal mapping from $D$ onto $U$ with $\omega(0)=0$ and $\omega^{\prime}(0)>0$, then

$$
\left|\omega^{\prime}(z)-1\right|<\frac{1}{2}, \quad z \in D
$$

and

$$
\frac{1}{2}(1-r)<1-\left|\omega\left(r e^{i t}\right)\right|<3(1-r), \quad 0<r<1, \quad|t|<\pi H .
$$

Let $q \geq 12$ be an integer and define

$$
f(z)=\sum_{n=1}^{\infty} w(z)^{q^{n}}, \quad z \in U
$$

i.e. $f(z)=f_{q}(\omega(z))$. Since the Bloch space is preserved under subordination (see e.g. $[17$, p. 35]), it follows that $f$ is a Bloch function. Then there exists a constant $C$ such that, for every $t$,

$$
\log ^{+}\left|f^{\prime}\left(r e^{i t}\right)\right| \leq \log \frac{1}{1-r}+C, \quad 0<r<1 .
$$

Now, (3.9) implies that

$$
\lim _{r \rightarrow 1} \frac{\log ^{+}\left|f^{\prime}\left(r e^{i t}\right)\right|}{\log \frac{1}{1-r}}=0, \quad \pi H<|t| \leq \pi
$$

and hence, by the dominated convergence theorem,

$$
\lim _{r \longrightarrow 1} \frac{1}{2 \pi} \int_{\pi H<|t| \leq \pi} \frac{\log ^{+}\left|f^{\prime}\left(r e^{i \ell}\right)\right|}{\log \frac{1}{1-\tau}} d t=0
$$


which, with (3.12), easily implies

$$
\underset{r \rightarrow 1}{\limsup } \frac{T\left(r, f^{\prime}\right)}{\log \frac{1}{1-r}} \leq H
$$

Let $r_{n}=1-q^{-n}$. Then, for $z=r_{n} e^{i t}$ with $|t| \leq \pi H$, we have

$$
\begin{aligned}
\left|\frac{\omega(z)}{\omega^{\prime}(z)} f^{\prime}(z)\right| & =\left|\sum_{k=1}^{\infty} q^{n} \omega(z)^{q^{n}}\right| \\
& \geq q^{n}|\omega(z)|^{q^{n}}-\sum_{k=1}^{n-1} q^{k}-\sum_{k=n+1}^{\infty} q^{k}|\omega(z)|^{q^{k}}=I-I I-I I I .
\end{aligned}
$$

Using (3.11). we obtain

$$
\left|\omega\left(r e^{i t}\right)\right|>3 r-2, \quad|t|<\pi H
$$

which implies that

$$
I=q^{n}|\omega(z)|^{q^{n}} \geq q^{n}\left(1-3 q^{-n}\right)^{q^{n}} .
$$

Hence, since $\left(1-3 j^{-1}\right)^{j} \geq e^{-4}(j \geq 12)$, we have

$$
I \geq e^{-4} q^{n}=e^{-4} \frac{1}{1-r_{n}}
$$

Now take $q$ so big that

$$
\frac{1}{q-1}<\frac{1}{4} e^{-4} \text { and }\left(3 e^{-1}\right)^{3} \frac{1}{q^{2}-1}<\frac{1}{4} e^{-4} .
$$

Then we have

$$
I I=\sum_{k=1}^{n-1} q^{k}<\frac{q^{n}}{q-1}=\frac{1}{q-1} \frac{1}{1-r_{n}}<\frac{1}{4} e^{-4} \frac{1}{1-r_{n}}
$$

and, by Schwarz's lemma and Lemma 1,

$$
\begin{aligned}
& I I I=\sum_{k=n+1}^{\infty} q^{k}|\omega(z)|^{q^{k}} \leq \sum_{k=n+1}^{\infty} q^{k}\left(1-q^{-n}\right)^{q^{k}} \\
& \quad \leq\left(3 e^{-1}\right)^{3} \frac{1}{q^{2}-1} q^{n}=\left(3 e^{-1}\right)^{3} \frac{1}{q^{2}-1} \frac{1}{1-r_{n}}<\frac{1}{4} e^{-4} \frac{1}{1-r_{n}}
\end{aligned}
$$

Hence $(3.14),(3.15),(3.16)$ and (3.17) show that

$$
\left|\frac{\omega(z)}{\omega^{i}(z)} f^{\prime}(z)\right| \geq \frac{1}{2} e^{-4} \frac{1}{1-r_{n}}, \quad z=r_{n} e^{i t}, \quad|t|<\pi H .
$$


Notice that $|\omega(z)|<1$ and that (3.10) implies that $\left|\omega^{\prime}(z)\right|>1 / 2$. Then (3.18) easily implies

$$
\left|f^{\prime}(z)\right| \geq \frac{1}{4} e^{-i} \frac{1}{1-r_{n}}, \quad z=r_{n} e^{i t}, \quad|t|<\pi H .
$$

It is clear that (3.19) shows that there exists a constant $C>0$ such that

$$
I_{2}\left(r_{n}, f^{\prime}\right) \geq \frac{C}{\left(1-r_{n}\right)^{2}}
$$

Since $I_{2}\left(r, f^{\prime}\right)$ is an increasing function of $r$, it follows that, for $r_{n} \leq r<r_{n+1}$,

$$
I_{2}\left(r, f^{\prime}\right) \geq I_{2}\left(r_{n}, f^{\prime}\right) \geq \frac{C}{\left(1-r_{n}\right)^{2}}=\frac{C}{q^{2}\left(1-r_{n+1}\right)^{2}}>\frac{C}{q^{2}} \frac{1}{(1-r)^{2}} .
$$

This proves (3.7).

Finally, (3.19) shows that for $n$ big enough,

$$
T\left(r_{n}, f^{\prime}\right) \geq \frac{1}{2 \pi} \int_{|t|<\pi H} \log ^{+}\left|f^{\prime}\left(r_{n} e^{i t}\right)\right| d t \geq H \log \frac{1}{4\left(1-r_{n}\right)}
$$

Consequently,

$$
\liminf _{r \rightarrow 1} \frac{T\left(r, f^{\prime}\right)}{\log \frac{1}{1-r}} \geq H
$$

which, with (3.13), implies (3.8),

We should remark that the condition $H>0$ is needed in Theorem 7 . In fact, it is a simple exercise to prove that if $f \in B$ and $T\left(r, f^{\prime}\right)=o\left(\log \frac{1}{1-r}\right)$, as $r \longrightarrow 1$, then $I_{2}\left(r, f^{\prime}\right)=o\left((1-r)^{-2}\right)$, as $r \longrightarrow 1$.

Pommerenke proved in [16, Th. 2] that if a Bloch function $f$ has radial limits almost everywhere on $|z|=1$ then it satisfies (1.8). Notice that the function $f$ constructed to prove Theorem 7 is in fact analytic on the set $\left\{e^{i t}: \pi H<\right.$ $|t| \leq \pi\}$ and consequently it has radial limits on a set of positive measure. The next result asserts that if a. Bloch furction $f$ satisfies this last condition then it satisfies (1.5).

Theorem 8. Let $f$ be a Bloch function having radial limits on a set of positive measure. Then

$$
\lim _{r \longrightarrow 1}\left(\log \frac{1}{1-r}-T\left(r, f^{\prime}\right)\right)=\infty
$$

Proof: Since a Bloch function is normal $[16$, p. 689], [1, p. 12] the concepts of radial limits and angular limits are equivalent for $f[15]$ (sce also [17, Th. 
9.3]). By Privalov's theorem [19, p. 320] these angular limits are finite almost everywhere. Hence, if we set

$$
E=\left\{t \in[-\pi, \pi]: f \text { has a finite angular limit at } e^{i t}\right\},
$$

then $|E|>0$.

Now,

$$
\begin{aligned}
& \log \frac{1}{1-r}-T\left(r, f^{\prime}\right)=\left(\frac{|E|}{2 \pi} \log \frac{1}{1-r}-\frac{1}{2 \pi} \int_{E} \log ^{+}\left|f^{\prime}\left(r e^{i t}\right)\right| d t\right) \\
& +\left(\frac{2 \pi-|E|}{2 \pi} \log \frac{1}{1-r}-\frac{1}{2 \pi} \int_{[-\pi, \pi]-E} \log ^{+}\left|f^{\prime}\left(r e^{i t}\right)\right| d t\right)=I+I I
\end{aligned}
$$

If $|E|=2 \pi$, then $I I=0$. Otherwise

$$
\begin{aligned}
I I & =\frac{2 \pi-|E|}{2 \pi}\left(\log \frac{1}{1-r}-\frac{1}{2 \pi-|E|} \int_{[-\pi, \pi]-E} \log ^{+}\left(\left|f^{\prime}\left(r e^{i t}\right)\right| d t\right)\right. \\
\geq \frac{2 \pi-|E|}{2 \pi}\left(\log \frac{1}{1-r}-\right. & \left.\frac{1}{2 \pi-|E|} \int_{[-\pi, \pi]-E} \log \left(\left|f^{\prime}\left(r e^{i t}\right)\right|+1\right) d t\right) \\
& =\frac{1}{2 \pi} \int_{(-\pi, \pi]-E} \log \frac{1}{(1-r)\left(\left|f^{\prime}\left(r e^{i t}\right)\right|+1\right)} d t
\end{aligned}
$$

Hence, since $f \in B$, there exists a constant $C$ such that

$$
I I \geq C, \quad 0<r<1 .
$$

Arguing as in the proof of [16, Th. 2] we obtain that

$$
(1-r)^{2} \int_{E}\left|f^{\prime}\left(r e^{i t}\right)\right|^{2} d t \longrightarrow 0, \text { as } r \longrightarrow 1,
$$

and then an argument like that used in the proof of Theorem 5(i) proves that $I \longrightarrow \infty$, as $r \longrightarrow 1$. This, with (3.21), shows that

$$
\log \frac{1}{I-r}-T\left(r, f^{\prime}\right) \longrightarrow \infty \text {, as } r \longrightarrow 1 \text {. }
$$

d) There are other questions that we could ask in this context. For instance, it seems natural to ask whether or not (1.5) is true if $f$ belongs to the closure of $H^{\infty}$ in $B$. The answer to this question is affirmative. Actually, it is easy to see that (1.8) is preserved under convergence in the Bloch norm and hence Theorem 2 and [16, Th. 2] show the following:

If $f \in C L_{B}(B \cap N)$ (the closure of $B \cap N$ in $B$ ) then $f$ satisfies (1.5).

Acknowledgments. I wish to thank the referee for his helpful comments, specially for his remarks about the gencrality of our results. Originally we just stated our results in the setting of Bloch functions and not in the more general framework of theorems 4 and 5 . 


\section{References}

1. J.M. Anderson, J.G. Clunie and Ch. Pommerenke, On Bloch functions and normal functions, J. Reine Angew. Math. 270 (1974), 12-37.

2. J.G. Clunie, On the derivative of a bounded function, Proc. London Math. Soc. (3) 14A (1965), 58-68.

3. P.L. Duren, "Theory of $H^{p}$ spaces," Academic Press, New York, 1970.

4. J.L. FERNÁNDEZ, On the coefficients of Bloch functions, J. London Math. Soc. (2) 29 (1984), 94-102.

5. J.L. FERNÁNDEZ, On the growth and coefficients of analytic functions, Ann. Math. 120 (1984), 505-516.

6. O. Frosrman, Sur les produits de Blaschke, Kungl. Fysiogr. Sällsk. $i$ Lund Förh. 12, 15 (1942), 169-182.

7. J.B. Garnetr, "Bounded Analytic Functions," Academic Press, New York, 1981.

8. D. Greta, Integral means and radial growth of Bloch functions, Math. Z. 195 (1987), 37-50.

9. D. Girela, On analytic functions with finite Dirichlet integral, Complex Voriables Theory Appl. 12 (1989), 9-15.

10. W.K. HAYMAN, "Research problems in function theory," London University Press, London, 1967.

11. W.K. HaYman, "Meromorphic functions," Oxford University Press, London, 1975.

12. W.K. Hayman, S.J. Patterson and CH. Pommerenke, On the coefficients of certain automorphic functions, Math. Proc. Combridge Phil. Soc. 82 (1977), 357-367.

13. P.B. Kennedy, A property of bounded regular functions, Proc. Roy. Irish Acad. 60, sect. A (1959), 7-14.

14. P.B. KENNEDY, On the derivative of a function of bounded characteristic, Quart. J. Math. Oxford (2) 15 (1964), 337-341.

15. O. LEHTO AND K.J. VIRTANEN, Boundary behaviour and normal meromorphic functions, Acta Math. 97 (1957), 47-65.

16. Ch. Pommerenke, On Bloch functions, J. London Math. Soc. (2), 2 (1970), 689-695.

17. CH. POMmerenke, "Univalent functions," Vandenhoeck und Ruprecht, Göttingen, 1975. 
18. E.J. SPECHT, Estimates on the mapping function and its derivatives in conformal mapping of nearly circular regions, Trans. Amer. Math. Soc. $\mathbf{7 1}$ (1951), 183-196.

19. M. TsujI, "Potential Theory in modern function theory," Chelsea Pu. Co., New York, 1975.

20. A. ZyGmund, "Trigonometric Series," Cambridge University Press, Cambridge, 1959.

\author{
Análisis Matemático \\ Facultad de Ciencias \\ Universidad de Málaga \\ 2907l Málaga \\ SPAIN
}

Primera versió rebuda el 15 de Febrer de 1990, darrera versió rebuda el 8 de Maig de 1990 